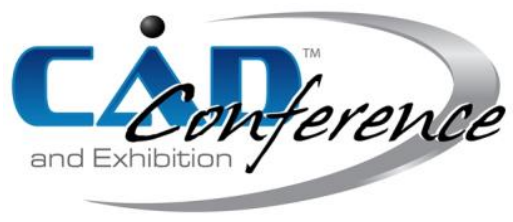

Title:

SHAPE TERRA: Industrial Feature Recognition based on Persistent Heat Signature

Authors:

Ramy Harik, harik@cec.sc.edu, University of South Carolina

Seung-Yeob Baek, bsy86@snu.ac.kr, Seoul National University

Barend-Jan van Bruchem, vanbruch@mailbox.sc.edu, University of South Carolina

Michel van Tooren, vantooren@cec.sc.edu, University of South Carolina

Keywords:

Shape matching, Persistence, Heat kernel, Feature recognition

DOI: 10.14733/cadconfP.2015.1-5

Introduction:

Shape recognition is a ubiquitous need. Pattern recognition on human models and objects is progressing steadily in many aspects of computer graphics such as garment design and biomedical engineering; but is still unexplored, immature and untapped in the mechanical/industrial world. Recognizing a shape for more then what it represents, embedding the manufacturing knowledge as well as processing techniques, will leverage shape recognition in industrial setup and would generate a real cost saving tool for industries. In this paper, we will present the Shape Terra project; we anticipate imbedding the shape with its meaning, being its: design parameters, manufacturing know-how and techniques, testing and quality, end of cycle treatment amongst others. The resulting tool looks at the typical mechanical feature identification research and attempts to profit from recent progress in nonmechanical components.

\title{
State of the Art:
}

Recent research in shape recognition for multimedia parts matching is well advancing. It uses the novel concept of investigating heat diffusion through the manifold [4]. Results are not applicable as is to mechanical parts because they do not hold acute points (corners), and if these exist they do not hold any meaning. The diverse fields of application were reviewed in [7]. They forward a qualitative overview of techniques classified into (a) Global feature-based; (b) manufacturing feature recognitionbased; (c) graph-based; (d) histogram based; (e) product information-based; and (f) 3D object recognition-based. Previous feature recognition techniques were based on geometrical identification of feature components followed by an interconnecting stage. This study overlooks traditional approaches and attempts to analyze heat persistence levels at different parts' clusters to identify and categorize potential mechanical features. One method to determine 3D shape similarity for feature recognition is the use of shape signatures. A shape signature is a concise representation of the shape but does not fully represent it. Instead of comparing the whole object, solely the signatures can be compared thus accelerating the matching technique. The challenge in shape retrieval is to have a descriptor that is invariant to as many transformations an object can experience, as well as difficulties such as scale variance and missing data [2]. Classification of deformable shapes using signatures based on geodesic distances leaves the identification process with limited robustness because geodesic distances are sensitive to local topological changes. To avoid using geodesic distances, deformation invariant descriptors such as the GPS embedding based on the combination of Laplace-Beltrami eigenvalues and eigenfunctions may be used [8]. Benchmarking of various feature detection and description algorithms show that heat kernel based feature detection and description algorithms perform best under many transformations [1]. Heat kernel descriptors involve solving the heat equation over the geometry of the part. The solution of the heat equation, the heat kernel, is related to the eigenvalues and 
eigenfunctions of the Laplacian. Geometrical information about part manifolds can be discovered using the Laplace operator together with the heat kernel signature (HKS). Multi-scale description is achieved by using the HKS on short and longer time periods to identify respectively local and global features [4]. The temperature distribution (TD) descriptor evaluates the temperature distribution evolution with time after the application of a unit heat at each vertex to retrieve the shape. Results show the effectiveness of this TD descriptor represented in a one-dimensional histogram for shape matching and retrieval [5]. Matching of partial or incomplete models cannot rely on global features so local features are needed. However, using a small time scale HKS to match local features increases the local variation in the HKS function values, thus making it more sensitive to noise. To overcome this problem and obtain an efficient pose-oblivious algorithm that can be used for all models, whether partial, incomplete or complete, persistent homology was introduced. Candidates for feature points are considered to be maxima of the HKS which persist beyond a given threshold, maxima that are not persistent are consequently eliminated. Research on descriptors of homology groups over arbitrary fields and research on reduction of computation effort by using Zigzag diagrams have made the advantage of persistent homology available in a much greater generality [3]. Experimental results show that persistent homology is effective in multi-scale shape matching [1].

\section{Main Idea:}

The broader goal of our initiative is to generate a neutral methodology of feature classification. Ideally, the user should be able to select a collection of points and run its analysis. The algorithm should return features that exhibit similar heat persistence features as well as identify the underlying mechanical property and dimensioning, eventually leading to a proper manufacturing plan. The study is conducted using MatLab ${ }^{\circledR}$ and Python without any CAD limitations. Recent work by the authors presented a novel approach to detect thin features - an abundant mechanical problem in aerospace parts [6]. The approach relies on combining persistence clustering and determining a thinness value. The current algorithm reorders all the identified features in a part from the most probable thin feature to the least probable. This will be presented to the process planner at the end of the design stage to confirm the selection and initiate particular manufacturing setup or to discard the selection. The analyst can use previously generated process plans and manufacturing programs from existing parts to rapidly estimate the manufacturing cost of the new part as well.
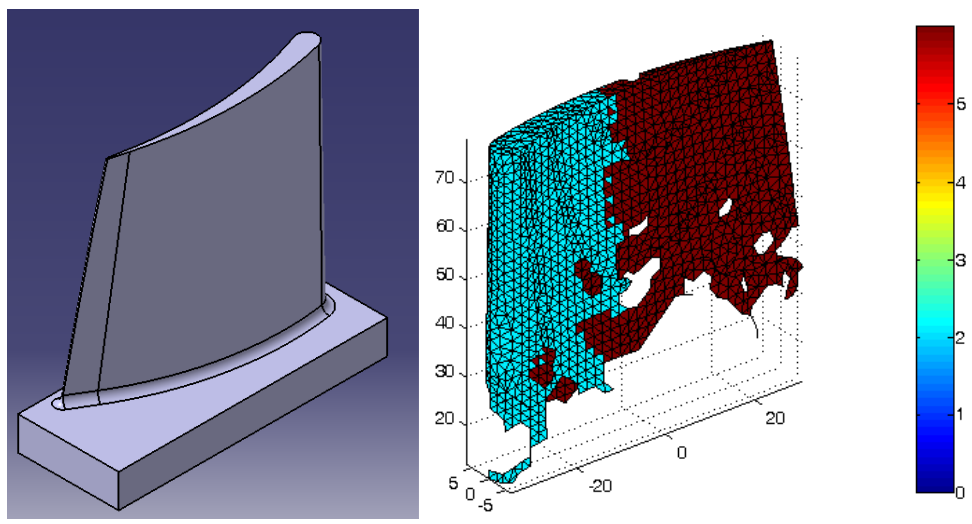

Fig. 1: Thinness detection on an airfoil component [6].

The approach consists of the following steps:

- $\quad$ Stage 1: Heat retention Signature

The heat retention of every point through time $R_{x}(t)$ is computed through the heat kernel equation $H_{t}(x, y)$, which is the solution of the partial differential heat equation $U(x, y)$ over the surface which can be expressed as an integral form. 


$$
\mathrm{U}(x, t)=1-\int_{\mathcal{M}} \mathrm{H}_{t}(x, y) U(y, 0) d y
$$

The heat kernel $H_{t}(x, y)$ can be decomposed in terms of eigenvalues and eigenfunctions.

$$
H_{t}(x, y)=\sum_{i=1}^{\infty} e^{-\lambda_{i} t} \phi_{i}(x) \phi_{i}(y)
$$

This leads to the heat retention of every point in time defined on a compact Riemannian 2-manifold $(\mathcal{M}, g)$ embedded in $\mathbb{R}^{3}$.

$$
\mathrm{R}_{x}(t)=1-\int_{\mathcal{M} \backslash D_{x}} \mathrm{H}_{t}(x, y) d y
$$

- $\quad$ Stage 2: Computing persistence level and value

Once heat retention is computed, the each point's resistance to loose heat is computed in terms of persistence level and value. Heat persistence level is defined as the time interval in which the heat value of a node persists above a minimum threshold $\mathrm{r}, P_{l}(x)=\frac{t_{i}}{\tau}$.

Persistence values are the integral of the heat function until the heat drops below a preset value or the area below the heat curve.

- $\quad$ Stage 3: Persistence Clustering

$$
\mathrm{P}_{v}(x)=\int_{0}^{t_{i}} R_{x}(t) d t
$$

Following stage 2, we set different persistence similarity levels and cluster subsets $\mathrm{K}$ accordingly. The algorithm runs from the highest head point $\mathrm{H}_{\mathrm{i}}$ (with highest persistence) and clusters with it all connected elements $\mathrm{P}_{\mathrm{i}}$ having a persistence higher than level $\mathrm{L}$.

$$
P_{i} \in K^{H_{i}} / \rho_{P_{i}} \in\left[L * \rho_{H_{i}} ; \rho_{H_{i}}\right]
$$

Clustering for low persistence similarity results in completely detected features with surrounding elements and noise components, not representative of any real feature. Clustering for higher similarity percentages results in partial feature clusters in elongated ring bands. An example of this step's result is shown in Figure 2.
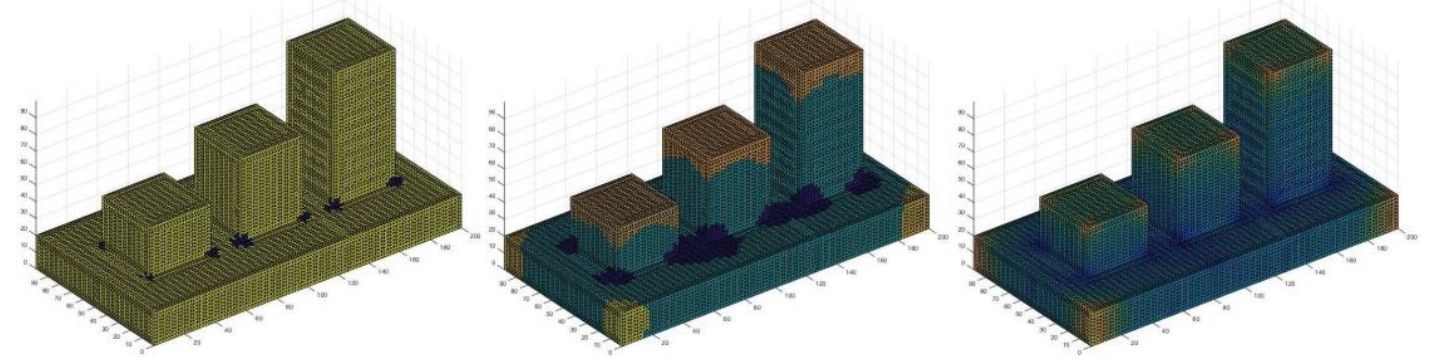

Fig. 2: Persistence clustering for $80 \%$ similarity.

- $\quad$ Stage 4: Multiscale filtering and enhancement

This stage is the most important recognition step. ShapeTerra attempts to refine previous clustering results by surfing across multiscale persistence similarities and to recognize optimal clustering. A multi-scale filtering technique is used to separate feature clusters. For this, a network graph-based method separating cold bodies from warm bodies is used. The concept is to recognize and split feature islands. A flood-fill method is used in this stage. An example of the results of this step is shown in Fig. 3.

\section{- Stage 5: Recognition and parameters identification}


Once features are recognized and characterized, we identify construction attributes such as cross-sectional area and height of protrusion/pocket orientation, as well as the orientation. Feature direction is obtained by Singular Value Decomposition to obtain the line that best fits the points present in the feature cluster. The cross-section is determined by projecting the nodes of the cluster on a plane normal to the direction vector.
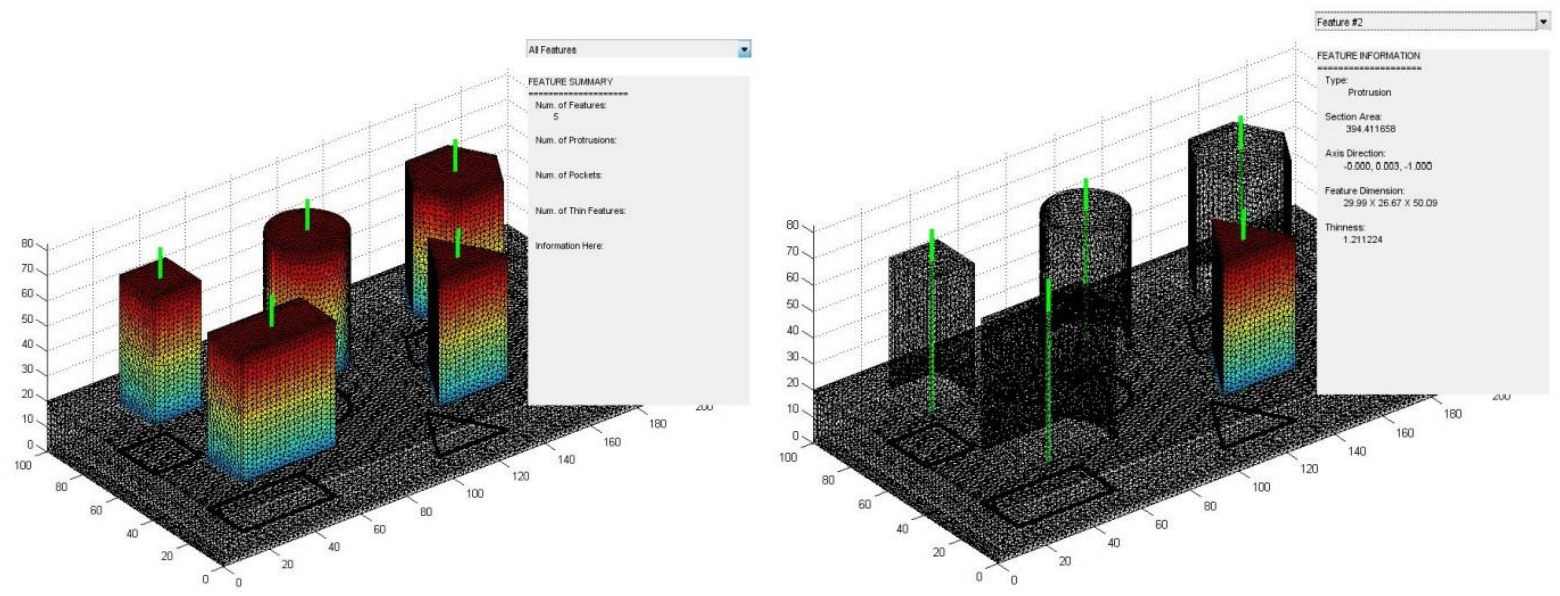

Fig. 3: Current snapshot of ShapeTerra.

Stage 1 of Shape Terra was enhanced since [6] and the error was reduced. Fig. 4 shows the comparison between the old results (in blue) and the new result (in red). Where the error average was at $4 \%$, it has now been reduced to less than $1 \%$. This increases the accuracy of the reported results.

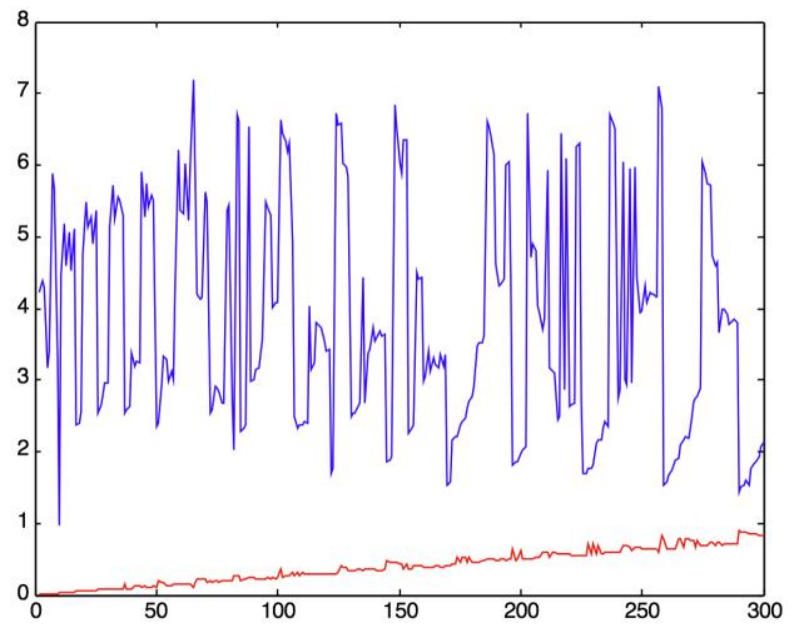

Fig. 4: Error comparing previous heat kernel coefficients (in blue) and new coefficients (in red) on a sphere model.

\section{Conclusions:}

No industrial solution exists today to recognize sub-parts attached to a part, how they should be obtained / manufactured - their intrinsic knowledge. All existing solutions rely heavily on a single operated CAD system and are often not available for research centers. When they exist, these solutions are based on variant and generative approaches and are not 'smart', in a sense that they proceed through a rigorous process of elimination. We propose, through Shape Terra, to solve this issue through a hybrid smart/generative approach; and to make it available online for web-CAD. 
This short paper presented ShapeTerra, an attempt at an online interactive research tool to explore shapes and recognize features and functions (such as the thinness presented in [6]). The authors will provide the backbone for recognition and, will make the tool available for industry to build their specific application requirement on top of it. Results so far show very promising capabilities of this methodology. Shape Terra is already in a stable phase of programming. Experiments are being conducted on automotive, aerospace and general parts. The reliability and selectiveness of the computation is also under scrutiny.

The current challenge will be to:

- $\quad$ Further optimize the shape descriptions for elements

- Advance the ongoing transition from an offline tool built in Matlab to an online tool functioning with Python

- Integrate a meshing pre-processing stage: the current tool requires a mesh as an input; future optimization involves having a simple coordinate file as input and dynamic reconstruction of an adaptive mesh

In the long run, the challenge will be to:

- Adapt the recognition to trade-specific knowledge (Additive Manufacturing, Subtractive Manufacturing ...)

- Enable the tool to run partial clouds of points (instead of a complete set)

- $\quad$ Add more specific functions, such as, manufacturing cost estimation, fixtures ...

\section{References:}

[1] Bronstein, A. M.; Bronstein, M. M.; Busto, B.; Castellani, U.; Crisani, M.; Falcidieno, B.; Guibas, L.J.; Kokkino, I.; Murino, V.; Ovsjanikov, M.; G. Patané, G.; I. Sipiran, I.; Spagnuolo, M.; Sun, L.: SHREC 2010: Robust Feature Detection and Description Benchmark, Eurographics Workshop on 3D Object Retrieval, 2010.

[2] Bronstein, M. M.; Kokkinos, I.: Scale-invariant heat kernel signatures for non-rigid shape recognition, Proceedings of the IEEE Computer Society Conference on Computer Vision and Pattern Recognition, 2010, 1704-1711. http://dx.doi.org/10.1109/CVPR.2010.5539838

[3] Carlsson, G.; Silva, V.; Morozov, D.: Zigzag Persistent Homology and Real-valued Functions, Proceedings of the Annual Symposium on Computational Geometry, 2009, 247-256. http://dx.doi.org/10.1145/1542362.1542408

[4] Dey, T.K.; Li, K.; Luo, C.; Ranjan, P.; Safa, I.; Wang, Y.: Persistent Heat Signature for Pose-oblivious Matching of Incomplete Models, Eurographics Symposium on Geometry Processing, 29(5), 2010. http://dx.doi.org/10.1111/j.1467-8659.2010.01763.x

[5] Fang, Y.; Sun, M.; Ramani, K.: Temperature Distribution Descriptor for Robust 3D Shape Retrieval, IEEE Computer Society Conference on Computer Vision and Pattern Recognition Workshops (CVPRW), 2011, 9-16. http://dx.doi.org/10.1109/CVPRW.2011.5981684

[6] Harik, R.; Durupt, A.; Khalifeh, J.; Eynard, B.; Recognition Of Thin Features Using Heat Diffusion, Proceedings of The Tenth International Symposium on Tools and Methods of Competitive Engineering, TMCE 2014, Budapest, Hungary, 2014, May 19-23.

[7] Iyer, N.; Jayanti, S.; Lou, K.; Kalyanaraman, Y.; Ramani, K.: Three-dimensional shape searching: state-of-the-art review and future trends, Computer Aided Design, 37(5), 2005, 509-530. http://dx.doi.org/10.1016/j.cad.2004.07.002

[8] Rustamov, R. M.: Laplace-Beltrami Eigenfunctions for Deformation Invariant Shape Representation, Eurographics Symposium on Geometry Processing, 2007. http://dx.doi.org/10.2312/SGP/SGP07/225-233 